\title{
Media Me: Body and Personal Media Interaction
}

\author{
Owen Noel Newton Fernando ${ }^{1}$, Imiyage Janaka Prasad Wijesena ${ }^{1}$, \\ Adrian David Cheok ${ }^{1}$, Ajith Parakum Madurapperuma ${ }^{2}$, \\ Lochandaka Ranathunga $^{2}$, Mei Gangwen ${ }^{1}$, Miyuru Dayarathna ${ }^{2}$, \\ Srinivasan Mariappan ${ }^{1}$, and Lee Rui Jie Jerome ${ }^{1}$ \\ ${ }^{1}$ Mixed Reality Lab, National University of Singapore, Singapore \\ ${ }^{2}$ Faculty of Information Technology, University of Moratuwa, Sri Lanka \\ contact@mixedrealitylab.org \\ http://www.mixedrealitylab.org
}

\begin{abstract}
Media Me" is a media interactive art work which comments on the bidirectional relationship between people and the media through the use of a realtime video mosaic. The elements of the video mosaic could be personal, cultural, historical, and educational. This research can be considered as a combination of creativity, art, and digital entertainment as well as an extension of personal media broadcasting. It comments on the growing trend of personal broadcasting and social media.
\end{abstract}

Keywords: Video mosaic, interactive media, human-media interaction.

\section{Introduction}

Television has been the mass media for broadcasting media content for a long time. However, developments in broadband internet and social networks have made it possible for individuals to use their own personal media as broadcast media. For example, "YouTube" allows individuals to submit personal videos for public viewing. From the previous concept of a few broadcast channels, now we have millions of channels broadcast by individuals. This has led Time Magazine to announce the person of the year in 2006 [1] as "you", to highlight the revolution in personal media that has developed.

As an artistic reflection on new personal media, Media Me is an interactive video installation that displays a captured image of a person as a video mosaic [2] 3] made of hundreds of videos. We literally turn the body into videos, which artistically represent the revolution in personal media. Videos are continuously arranged in realtime to form a mosaic representation of the background to provide meaningful contents, such as cultural and historical media. When no image is captured by the system, Media Me activates and reflects the media itself by creating a mosaic of cultural and historical content.

Media Me can be considered as a new form of personal media where a person can create and broadcast her own customized contents as image elements. For the current version of Media Me, we have used religion, cultural, and historical movies of Sri Lanka to create a meaningful video Mosaic. This system can also 


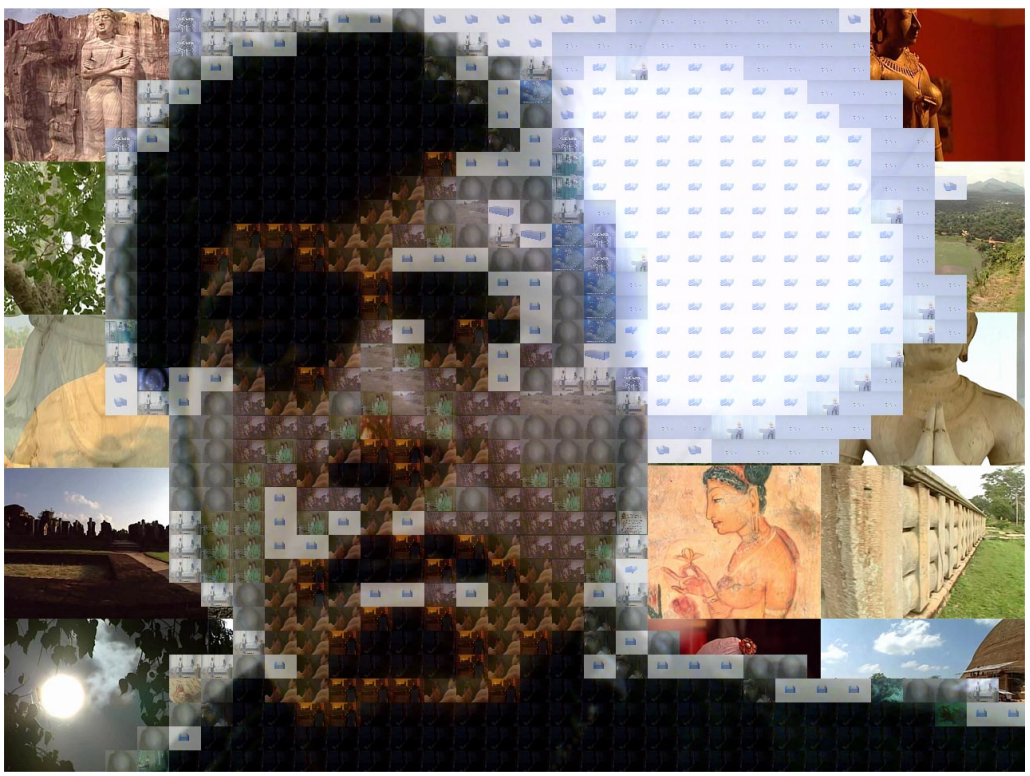

(a) National Heritage of Sri Lanka

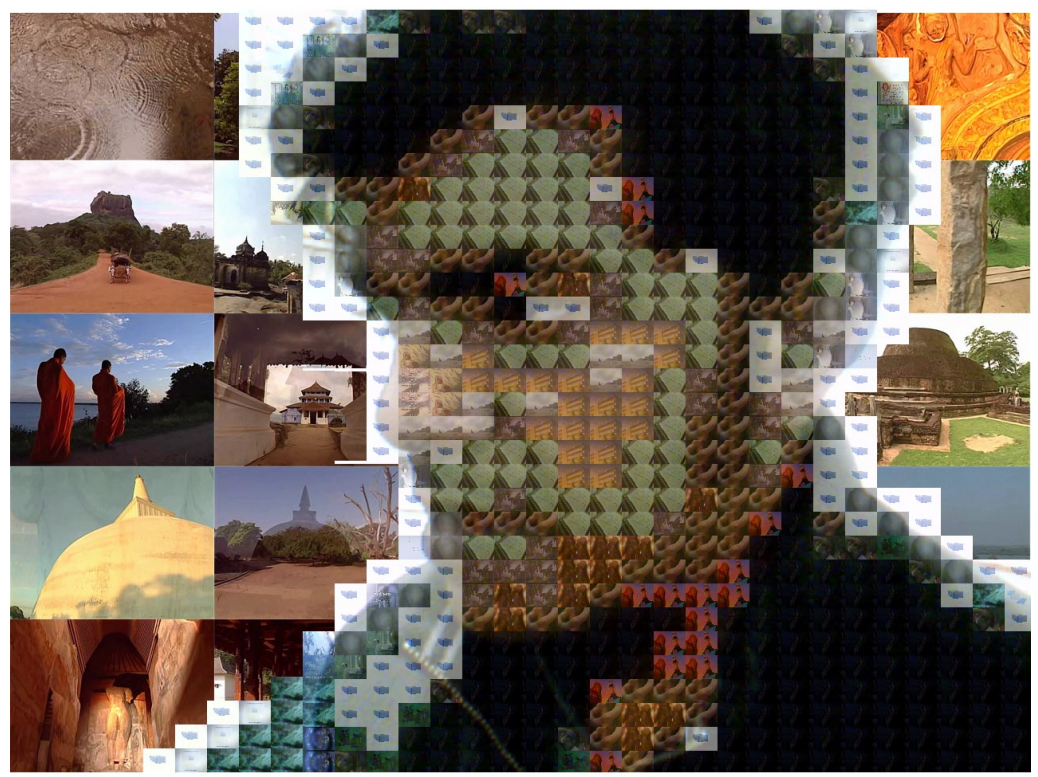

(b) Buddhism in Sri Lanaka

Fig. 1. Mosaic created by the system 
be used for educational purposes in an interactive way, for example exploring national heritage of Sri Lanka. We are also extending the system for various other cultures.

In Figure 1(a) the face (foreground) and the background mosaic are constructed with video clips showing national heritage of Sri Lanka. Similarly, Figure 1(b) shows mosaic constructed with videos of Buddhism in Sri Lanka. The background videos are randomly selected and arranged by the system. The system analyzes each area of the foreground and selects a video clip that can substitute that area. As shown in the Figure 1, some level of colour correction is applied to the foreground video clips to attain a more natural look and feel.

\section{System Overview}

The image of the person, who stands in front of the blue screen, is captured by the camera. In the system initialization process the average colour of the background is computed and it is used to remove the background from the extracted video frame. The foreground is segmented to rectangular areas and average colour of each of them is calculated. The average colour is used to find a matching video clips from the video database. The video clips in the database are pre-analyzed and organized based on their average color. Since the system has only a finite number of videos, some amount of colour correction is applied to the selected video clips in order to attain the realistic look and feel. The background of the original video is removed and replaced with larger tiled set of videos. These videos are randomly selected from a video database. Finally, the background and the foreground are combined to create the mosaic. An electronic projector projects the final video mosaic onto a large screen right before the person. The full system architecture is shown in Figure 2.

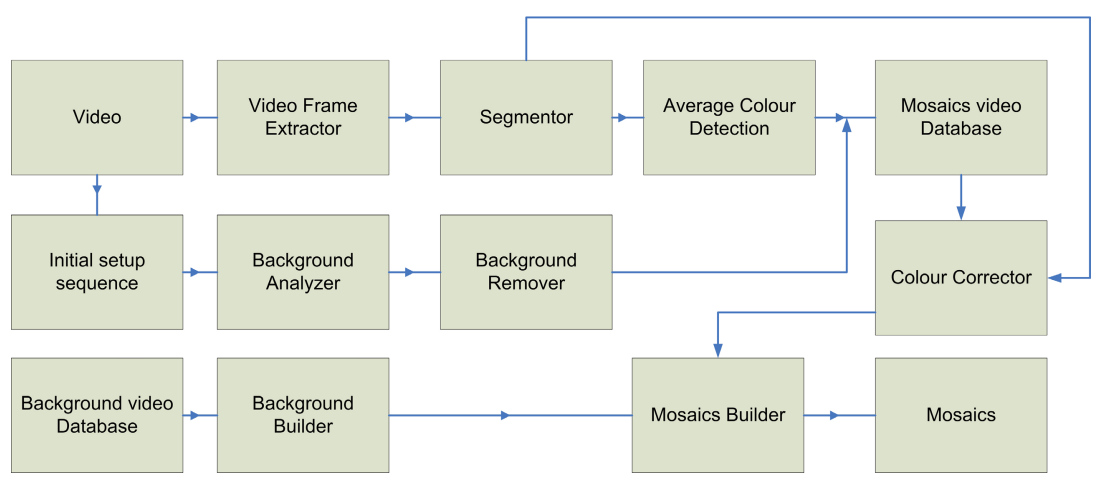

Fig. 2. Media Me: System Architecture 


\section{Conclusion}

Computing technologies are increasingly being used to support new forms of entertainment and creativity. Creativity, art, and digital entertainment systems provide futuristic new media forms. Media $\mathrm{M} 1$ is a media interactive art work which comments on the bidirectional relationship between people and the media through the use of a realtime video mosaic. It also provides the means to educate the masses while entertaining them. This will also bring new ways of communication between people and media, and new forms of social, educational, and cultural interaction.

Acknowledgments. Media Me is funded by Singapore Science Center. This project is installed in the iSpace at Singapore Science center to allow visitors to obtain a novel experience in entrainment and social communication.

\section{References}

1. Time Magazine Website (December 13, 2006) http://www.time.com/time/magazine/article/0, 9171, 1569514,00.html

2. Mackay, W., Pagani, D.: Video mosaic: laying out time in a physical space. In: MULTIMEDIA '94. Proc. of the second ACM Int. Conf. on Multimedia, California, USA, pp. 165-172 (1994)

3. Klein, A.W., Grant, T., Finkelstein, A., Cohen, M.F.: Video mosaics. In: NPAR '02. Proc. of the 2nd Int. symposium on Non-photorealistic animation and rendering, Annecy, France, pp. 21-29 (2002)

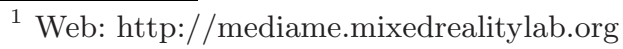

